\title{
Mitochondrial DNA haplogroup $T$ is associated with coronary artery disease and diabetic retinopathy: a case control study
} Barbara Kofler*1, Edith E Mueller ${ }^{1}$, Waltraud Eder ${ }^{1}$, Olaf Stanger ${ }^{2}$, Richard Maier ${ }^{3}$, Martin Weger ${ }^{3}$, Anton Haas ${ }^{3}$, Robert Winker ${ }^{4}$, Otto Schmut ${ }^{3}$, Bernhard Paulweber ${ }^{5}$, Bernhard Iglseder 6 , Wilfried Renner ${ }^{7}$, Martina Wiesbauer ${ }^{1}$, Irene Aigner ${ }^{2}$, Danijela Santic ${ }^{1}$, Franz A Zimmermann ${ }^{1}$, Johannes A Mayr ${ }^{1}$ and Wolfgang Sperl ${ }^{1}$

Address: ${ }^{1}$ Department of Pediatrics, University Hospital Salzburg, Paracelsus Medical University, Salzburg, Austria, ${ }^{2}$ Department of Cardiac Surgery, University Hospital Salzburg, Paracelsus Medical University, Salzburg, Austria, ${ }^{3}$ Department of Ophthalmology, Medical University Graz, Graz, Austria, ${ }^{4}$ Department of Occupational Medicine, Medical University of Vienna, Vienna, Austria, ${ }^{5}$ Department of Internal Medicine, University Hospital Salzburg, Paracelsus Medical University, Salzburg, Austria, ${ }^{6}$ Department of Geriatrics, University Hospital Salzburg, Paracelsus Medical University, Salzburg, Austria and ${ }^{7}$ Clinical Institute of Medical and Chemical Laboratory Diagnostics, Medical University Graz, Graz, Austria

Email: Barbara Kofler* - b.kofler@salk.at; Edith E Mueller - ed.mueller@salk.at; Waltraud Eder - w.eder@salk.at; Olaf Stanger - o.stanger@salk.at; Richard Maier - richardmaier@chello.at; Martin Weger - martin.weger@meduni-graz.at; Anton Haas - anton.haas@uni-graz.at; Robert Winker - robert.winker@meduniwien.ac.at; Otto Schmut - otto.schmut@meduni-graz.at; Bernhard Paulweber - b.paulweber@salk.at; Bernhard Iglseder - b.iglseder@salk.at; Wilfried Renner - wilfried.renner@meduni-graz.at; Martina Wiesbauer - martina.wiesbauer@sbg.ac.at; Irene Aigner - irene.aigner@gmx.at; Danijela Santic - danijela@gmx.at; Franz A Zimmermann - f.zimmermann@salk.at; Johannes A Mayr - h.mayr@salk.at; Wolfgang Sperl - w.sperl@salk.at

* Corresponding author

Published: 21 April 2009

BMC Medical Genetics 2009, 10:35 doi:10.1 186/1471-2350-10-35
Received: I October 2008

Accepted: 21 April 2009

This article is available from: http://www.biomedcentral.com/I47I-2350/I0/35

(C) 2009 Kofler et al; licensee BioMed Central Ltd.

This is an Open Access article distributed under the terms of the Creative Commons Attribution License (http://creativecommons.org/licenses/by/2.0), which permits unrestricted use, distribution, and reproduction in any medium, provided the original work is properly cited.

\begin{abstract}
Background: There is strong and consistent evidence that oxidative stress is crucially involved in the development of atherosclerotic vascular disease. Overproduction of reactive oxygen species (ROS) in mitochondria is an unifying mechanism that underlies micro- and macrovascular atherosclerotic disease. Given the central role of mitochondria in energy and ROS production, mitochondrial DNA (mtDNA) is an obvious candidate for genetic susceptibility studies on atherosclerotic processes. We therefore examined the association between mtDNA haplogroups and coronary artery disease (CAD) as well as diabetic retinopathy.
\end{abstract}

Methods: This study of Middle European Caucasians included patients with angiographically documented CAD ( $n=487)$, subjects with type 2 diabetes mellitus with $(n=149)$ or without $(n=$ 78) diabetic retinopathy and control subjects without clinical manifestations of atherosclerotic disease $(n=1527)$. MtDNA haplotyping was performed using multiplex PCR and subsequent multiplex primer extension analysis for determination of the major European haplogroups. Haplogroup frequencies of patients were compared to those of control subjects without clinical manifestations of atherosclerotic disease.

Results: Haplogroup $T$ was significantly more prevalent among patients with CAD than among control subjects $(14.8 \%$ vs $8.3 \%$; $p=0.002)$. In patients with type 2 diabetes, the presence of 
diabetic retinopathy was also significantly associated with a higher prevalence of haplogroup $\mathrm{T}$ ( $12.1 \%$ vs $5.1 \%$; $p=0.046)$.

Conclusion: Our data indicate that the mtDNA haplogroup $T$ is associated with CAD and diabetic retinopathy in Middle European Caucasian populations.

\section{Background}

Mitochondria are the primary source of endogenous reactive oxygen species (ROS), generated through oxidative phosphorylation (OXPHOS) as a by-product of ATP synthesis. It is a generally accepted principle that in normal cells, ROS form a regular part of diverse redox signalling pathways, whereas in many types of chronic and cardiovascular diseases, ROS production exceeds antioxidant capacity with damaging effects on cell function and structure [1]. Therefore, mitochondrial dysfunction has been implicated in the pathogenesis of several diseases characterised by free radical-induced damage. Given the central role of mitochondria in energy and ROS production, mitochondrial DNA (mtDNA) is an obvious candidate for genetic susceptibility studies on atherosclerotic processes. The extranuclear mitochondrial genome is highly polymorphic and this variation could contribute to the range of complex traits in energy metabolism. Four of the enzymatic complexes that constitute the oxidative phosphorylation system (OXPHOS) are partially encoded by the mtDNA. Rare pathogenic mutations of the mtDNA, that either impair mitochondrial protein synthesis or impair proteins encoded by the mtDNA have been associated with more than 70 human diseases [2].

During evolution, a number of mutations have accumulated in the mtDNA [3]. Mutations acquired during evolution have subdivided the human population into a number of discrete, region specific, mitochondrial clades or haplogroups. MtDNA haplogroups are defined on the basis of specific single nucleotide polymorphisms (SNPs) scattered throughout the mitochondrial genome. In populations of European ancestry, nine such haplogroups with frequencies of at least 1\% have been described [4]. Emerging evidence suggests that different mtDNA haplogroups are associated with subtle differences in OXPHOS capacity and the generation of ROS $[5,6]$. Recent evidence suggests that mtDNA haplogroups have functional consequences, being linked to longevity [7], sperm motility [5], certain types of cancer [8] and affecting the risk of individuals developing specific late-onset neurodegenerative diseases [9].

Diabetic retinopathy is caused by alterations in the retinal microvasculature leading to breakdown of the blood-retina barrier and pathological angiogenesis [10] and is one of the primary causes of visual loss worldwide [11]. Diabetic retinopathy and CAD are two vascular complications of type 2 diabetes mellitus, representing examples of microangiopathy and macroangiopathy, respectively. Retinopathy is also an early and frequent sign of other vascular complications and is strongly associated with the development of CAD $[12,13]$. This indicates that CAD and diabetic retinopathy share common risk factors and pathological mechanisms, such as diabetes and inflammation, which both exhibit increased ROS production $[14,15]$.

It has been shown that oxidative stress due to disturbance in the balance between production of ROS and antioxidant defence plays a vital role in the pathogenesis of coronary atherosclerosis and its complications [15].

We aimed to investigate the association of mtDNA haplogroups with CAD as well as diabetic retinopathy.

\section{Methods \\ Patients and control subjects}

Data from 2352 Caucasian subjects enrolled in three surveys in central and southern Austria were analysed (Table $1)$. The study was conducted according to the Austrian Gene Technology Act and complied with the Declaration of Helsinki. All subjects gave written informed consent before entering the study.

Patients with heart diseases $(n=598)$ were recruited in Salzburg and Graz. Four hundred and eighty-seven individuals had angiographically documented CAD with at least one of the main coronary arteries showing $\geq 50 \%$ stenosis. CAD was angiographically excluded in 111 patients with valvular heart disease.

The study population of patients with diabetes $(n=227)$ was recruited at the University Hospital of Graz and consisted of patients with $(n=149)$ and without diabetic retinopathy $(n=78)$. Presence and severity of diabetic retinopathy were assessed by fundus examination. Diabetic retinopathy was graded according to the modified "Early Treatment Diabetic Retinopathy Study" (ETDRS) retinopathy scale as no apparent retinopathy, non-proliferative, and proliferative diabetic retinopathy.

The control population consisted of 1527 unrelated individuals as previously described in detail [16]. Briefly, the cohort comprised 988 men between 39 and 66 years of age and 539 women between 39 and 67 years of age who 
Table I: Characteristics of the study populations.

\begin{tabular}{|c|c|c|c|c|c|}
\hline & \multirow{2}{*}{$\begin{array}{l}\text { Controls } \\
n=1527\end{array}$} & \multicolumn{2}{|c|}{ Patients with heart disease } & \multicolumn{2}{|c|}{ Patients with diabetes } \\
\hline & & $\begin{array}{c}C A D \\
n=487\end{array}$ & $\begin{array}{c}\text { Valvular Heart Disease } \\
\qquad \mathrm{n}=\mathrm{I} \mathrm{I} \mathrm{I}\end{array}$ & $\begin{array}{l}\text { No Retinopathy } \\
\qquad n=78\end{array}$ & $\begin{array}{c}\text { Retinopathy } \\
n=149\end{array}$ \\
\hline Mean (SD*) age (years) & $51.5(6.1)$ & $63.4(11.3)$ & $64.9(12.9)$ & $76.9(9.8)$ & $7 I .1$ (II.2) \\
\hline Male (\%) & 64.7 & 76.4 & 54.1 & 47.4 & 36.9 \\
\hline Mean (SD*) BMI† $\left(\mathrm{kg} / \mathrm{m}^{2}\right)$ & $26.6(4.1)$ & $27.3(3.7)$ & $26.3(4.5)$ & $27.5(4.5)$ & $27.7(5.3)$ \\
\hline History of myocardial infarction (\%) & 0 & 50.5 & 0 & 7.7 & 14.8 \\
\hline Diagnosis of diabetes (\%) & 0 & 25.5 & 16.2 & 100 & 100 \\
\hline Hypertension (\%) & 13.7 & 67.6 & 59.5 & 75.6 & 71.8 \\
\hline Nonsmoker (\%) & 65.3 & 48.7 & 72.4 & n.a. $\ddagger$ & n.a. $\ddagger$ \\
\hline Former smoker (\%) & 13.5 & 37.5 & 14.3 & n.a. $\ddagger$ & n.a. $\ddagger$ \\
\hline Current smoker (\%) & 21.2 & 13.8 & 13.3 & n.a. $\ddagger$ & n.a. $\ddagger$ \\
\hline
\end{tabular}

*SD: standard deviation; †BMI: body mass index; $\ddagger$ n.a. = not available.

were recruited in the Salzburg Atherosclerosis Prevention Program (SAPHIR) [16]. Exclusion criteria for participation in this study were a history of CAD, heart failure, cerebrovascular disease, peripheral vascular disease, haemodynamically relevant heart valve disease, chronic disease (of liver or kidney, autoimmune disorders, malignant cancer, haematologic disorders, endocrinopathies, diabetes mellitus) and morbid obesity. Laboratory methods have been described previously [16]. The haplogroup frequency in 1172 individuals of the control cohort was reported [17]. The study was approved by the Ethics Committee of the Medical Association of Salzburg.

\section{MtDNA analysis}

Venous blood was collected in $5 \mathrm{ml}$ EDTA tubes; total DNA was isolated with a Nucleospin Blood Kit (Macherey-Nagel) and stored at $4^{\circ} \mathrm{C}$. A hierarchical system for mtDNA haplogrouping that combines multiplex PCR amplifications, multiplex single-base primer extensions, and capillary-based electrophoretic separation for analysing ten haplogroup-diagnostic mitochondrial SNPs (mtSNPs) was used to determine the haplogroup distribution of the most common European haplogroups H, U, J, T, K, I, V, W and X, as described previously [17]. Haplogroups that could not be assigned to one of the nine major European haplogroups by the SNP combination were designated as "others". From the 2387 subjects investigated, genotyping failed in $0.21 \%$ of the samples $(n=5)$. PCR amplification failed due to degradation of DNA in $1.26 \%$ of samples $(\mathrm{n}=30)$.

\section{Statistical analysis}

Frequencies of all mitochondrial haplogroups in CAD patients and in controls were tested for independency using Pearson chi-square statistics and Fisher's exact test as appropriate. Similarly, frequencies of mitochondrial haplogroups were compared between patients with heart disease other than CAD and controls. For further analysis only low frequency haplogroups $(\mathrm{K}, \mathrm{W}, \mathrm{V}, \mathrm{I}, \mathrm{X})$ were excluded from further analyses. Age and gender adjusted odds ratios and 95\% confidence intervals were then calculated to express the strength of the associations between the haplogroups and phenotypes. The following variables were also considered as potential confounders and tested in logistic regression models: hypertension (yes/no), body mass index (BMI), and smoking (never, former smoker, current smoker). Covariates that had a significance level of less than 0.1 were retained to create the bestfitting model based on Akaike's information criterion. If one category of an independent variable had a significance of 0.1 or less, all parts of the categorical variable were retained in the best-fitting model. P-values were corrected for multiple comparison by Bonferroni analysis (required significance level $=0.05 /$ number of comparisons), leading to a new required significance level of $<0.008$ [number of comparisons $=6(3 \times 2)$ for 3 haplogroups compared to haplogroup $\mathrm{H}$ and to two diseases (CAD and Valvular Heart Disease)].

The same methods as described above were applied to calculate associations between mitochondrial haplogroups and the presence of retinopathy among patients with diabetes. Sex-adjusted odds ratios and 95\% confidence intervals were then tested for the potential confounding effect of age at onset of diabetes (years), duration of diabetes (months), levels of HbA1c (\%) measured at the time of recruitment, medication (insulin, oral antidiabetics), history of myocardial infarction (yes/no), and hypertension (yes/no). The best-fitting model was created in the same manner as for the heart disease population. P-values were corrected for multiple comparison by Bonferroni analysis (required significance level $=0.05 /$ number of comparisons), leading to a new required significance level of $<0.017$ (number of comparisons = 3 for 3 haplogroups compared to haplogroup $\mathrm{H}$ ).

All analyses were performed using SPSS 15.0 student version (SPSS GmbH Software, 80339 Munich, Germany) 
and Stata/SE 10.0 (Stata Corporation, College Station, TX, USA).

\section{Results}

Clinical characteristics of patients and controls are shown in Table 1. All nine European haplogroups were observed in our sample, as expected (Table 2). Frequencies in the control group did not differ significantly from those previously reported for neighbouring European Caucasian populations $[17,18]$.

Frequencies of mitochondrial haplogroups in patients with CAD differed significantly from those in healthy controls. The frequency of haplogroup T in controls was $8.3 \%$ and in patients with CAD $14.8 \%$. After adjustment for sex and age we calculated an OR (95\% CI) of 2.36 (1.52$3.65)$ for the association of haplogroup T with CAD. Further adjustment for possible confounders revealed that the strong association between haplogroup $\mathrm{T}$ and CAD was not explained by differences in age, sex, hypertension, body mass index, or smoking status (Table 3 ). Even after adjustment for multiple comparisons by Bonferroni correction the p-value remained significant. None of the other haplogroups showed a significant association with CAD (Table 3). Haplogroup frequencies were very similar in the two study centres, Salzburg and Graz. Nearly identical associations were seen when the analyses were stratified according to the two study centres from which the patients had been recruited. The age- and sex-adjusted OR ( $95 \% \mathrm{CI})$ for the association between CAD and mitochondrial haplogroup $\mathrm{T}$ was significant in patients recruited in Graz [ $\mathrm{n}=195 ;$ OR $2.22(1.36-3.63) ; \mathrm{p}=0.001]$ and those recruited in Salzburg [ $\mathrm{n}=292 ;$ OR $2.36(1.14-4.88) ; \mathrm{p}=$ 0.021]. In contrast, no difference in the frequencies of mitochondrial haplogroups was seen between patients with valvular heart disease with documented absence of CAD and healthy controls (Table 3 ).

We next compared the frequencies of the mitochondrial haplogroups between patients with diabetes with retinop- athy and patients with diabetes without retinopathy. Haplogroup $T$ was significantly more prevalent in patients with diabetic retinopathy than in patients without that condition $(12.1 \%$ vs $5.1 \%$; Tables 2,4$)$. There was a positive association between haplogroup $\mathrm{T}$ and retinopathy among patients with diabetes, which became significant when the best-fitting model was created (Table 4). After adjustment for multiple comparisons by Bonferroni correction the association was no longer significant, most likely due to the small sample size. Subgroup analysis revealed no significant differences in DNA haplogroups between patients with and without proliferative diabetic retinopathy $(\mathrm{n}=75$; Pearson chi-square $3.4, \mathrm{df} 4, \mathrm{p}=$ 0.493). Also, no trend of haplogroup $T$ with severity of retinopathy was observed as can be derived from the frequencies of haplogroup $\mathrm{T}$ in non-proliferative retinopathy $(13.3 \%)$ and proliferative retinopathy $(10.8 \%)$.

\section{Discussion}

Our data show an association of mtDNA haplogroup T with coronary artery disease and diabetic retinopathy. Mitochondrial dysfunction has been implicated in the pathogenesis of several diseases characterised by free radical-induced damage [19-21]. Genetic association of specific mtDNA haplogroups has been demonstrated with aging and multifactorial age-related diseases like Alzheimer's disease, multiple sclerosis and cancer [22]. Although the precise mechanisms underlying the associations of mtDNA haplogroups with multifactorial agerelated diseases including CAD and diabetic retinopathy remain speculative, haplogroup-specific differences in free radical production during OXPHOS may be one such mechanism. Thus, presence of haplogroup $\mathrm{T}$ might be associated with increased oxidative stress or increased susceptibility to oxidative stress. Individuals carrying haplogroup $\mathrm{T}$ might be more vulnerable to oxidative damage than carriers of other haplogroups.

While intracellular ROS serve mainly for host defence against infectious agents, redox-sensitive signal transduc-

Table 2: Frequencies (\%) of mitochondrial haplogroups in cases and controls.

\begin{tabular}{|c|c|c|c|c|c|}
\hline \multirow[t]{2}{*}{ Haplogroup } & \multirow[t]{2}{*}{ Controls } & \multicolumn{2}{|c|}{ Patients with heart diseases } & \multicolumn{2}{|c|}{ Patients with diabetes } \\
\hline & & CAD & Valvular Heart Disease & No Retinopathy & Retinopathy \\
\hline & $\mathrm{n}=1527$ & $n=487$ & $\mathrm{n}=1 \mathrm{II}$ & $n=78$ & $n=149$ \\
\hline $\mathrm{H}$ & 43.6 & 37.8 & 46.0 & 52.5 & 43.6 \\
\hline U & 15.5 & 15.0 & 16.2 & 11.5 & 14.8 \\
\hline J & 11.4 & 10.9 & 12.6 & 5.1 & 10.7 \\
\hline $\mathrm{T}$ & 8.3 & 14.8 & 9.0 & 5.1 & 12.1 \\
\hline K & 5.2 & 3.1 & 0.9 & 3.9 & 3.4 \\
\hline W & 2.1 & 1.8 & 1.8 & 0.0 & 2.0 \\
\hline V & 1.8 & 3.1 & 0.9 & 2.6 & 2.0 \\
\hline I & 1.0 & 1.2 & 1.8 & 1.3 & 0.7 \\
\hline $\mathrm{x}$ & 1.3 & 3.1 & 1.8 & 3.9 & 2.0 \\
\hline others & 9.8 & 9.2 & 9.0 & 14.1 & 8.7 \\
\hline
\end{tabular}


Table 3: Odds ratios $(\mathrm{OR})$ and $95 \%$ confidence intervals $(\mathrm{CI})$ for the association between mitochondrial haplogroup and CAD and Valvular Heart Disease, respectively.

\begin{tabular}{|c|c|c|c|c|c|}
\hline & Haplogroup & OR* $(95 \% \mathrm{Cl})$ & $\mathrm{p}$-value & $\mathrm{aOR}^{\dagger}(95 \% \mathrm{Cl})$ & $\mathrm{p}$-value \\
\hline \multicolumn{6}{|l|}{ Coronary artery disease } \\
\hline & $\mathrm{H}$ & I (ref) & & I (ref) & \\
\hline & $U$ & $1.06(0.69-1.61)$ & 0.791 & $1.18(0.72-1.93)$ & 0.504 \\
\hline & $J$ & $0.92(0.58-1.47)$ & 0.738 & $1.09(0.63-1.89)$ & 0.766 \\
\hline & $\mathrm{T}$ & $2.36(1.52-3.65)$ & $<0.0005^{\ddagger}$ & $2.33(1.37-3.98)$ & $0.002 \ddagger$ \\
\hline \multicolumn{6}{|l|}{ Valvular Heart disease } \\
\hline & $\mathrm{H}$ & I (ref) & & & \\
\hline & $U$ & $1.05(0.50-2.17)$ & 0.903 & & \\
\hline & $J$ & $0.96(0.44-2.14)$ & 0.930 & & \\
\hline & $\mathrm{T}$ & $1.24(0.52-2.96)$ & 0.626 & & \\
\hline
\end{tabular}

*Adjusted for age and sex (CAD $n=382$, Valvular heart disease $n=93$, controls $n=1203)$; tadjusted for age, sex, smoking, hypertension, and body mass index $(C A D n=345$, controls $n=1095)$; łBonferroni-corrected statistical significance: $p<0.05 / 3 \times 2=<0.008$.

tion, and other cellular processes, the extracellular release of ROS may damage surrounding tissues, potentially promoting inflammatory processes $[23,24]$. ROS are involved in aging and many diseases such as atherosclerosis, cancer, diabetes mellitus, neurological degeneration, and tumor invasion. There is strong evidence that inflammation also plays a major role in the etiology and progression of diabetic retinopathy [14]. Inflammation is an integral part not only of CAD but also of other complex diseases like multiple sclerosis, where an association of mtDNA haplogroups has been reported [25]. Enhanced formation of ROS may affect four fundamental mechanisms that contribute to atherogenesis, namely: oxidation of low density lipoprotein (LDL), endothelial dysfunction, vascular smooth muscle cell growth, and monocyte migration [21]. Interestingly, we did not find an association of haplogroup $\mathrm{T}$ to markers of inflammation like Creactive protein (CRP) and LDL (data not shown).

Previous exact analysis of specific differences in the mtDNA haplogroups revealed that haplogroup T is generally associated with a number of polymorphisms in the non-coding control region $(16126,16294)$, mitochondrial ribosomal RNAs $(709,1888)$, mitochondrial transfer

Table 4: Odds ratios (OR) and $95 \%$ confidence intervals $(\mathrm{Cl})$ for the association between mitochondrial haplogroup and retinopathy in patients with diabetes $(n=179)$.

\begin{tabular}{lllll}
\hline Haplogroup & $\mathrm{OR}^{*}(95 \% \mathrm{Cl})$ & $\mathrm{P}$-value & $\mathrm{aOR}+(95 \% \mathrm{Cl})$ & $\mathrm{P}$-value \\
\hline $\mathrm{H}$ & $\mathrm{I}($ ref $)$ & & $\mathrm{I}($ ref $)$ & \\
$\mathrm{U}$ & $\mathrm{I} .54(0.64-3.69)$ & 0.330 & $\mathrm{I} .92(0.72-5.07)$ & 0.190 \\
$\mathrm{~J}$ & $2.59(0.8 \mathrm{I}-8.34)$ & 0.110 & $2.04(0.50-8.36)$ & 0.324 \\
$\mathrm{~T}$ & $2.97(0.93-9.48)$ & 0.065 & $3.60(1.02-12.68)$ & $0.046 \ddagger$ \\
\hline
\end{tabular}

*Adjusted for sex; tadjusted for sex, history of myocardial infarction, levels of $\mathrm{HbAlc}$ and therapy with oral antidiabetics; $¥$ Bonferronicorrected statistical significance $p<0.05 / 3=<0.017$.
RNAs $(10463,15928)$, several SNPs not changing amino acids in the corresponding proteins, and a polymorphism at position 4917 leading to a change of asparagin to aspartate in the ND2 protein [26]. All these SNPs could contribute to altered OXPHOS performance and ROS production. This hypothesis was substantiated by RuizPesini et al., who have shown that sperm cells of individuals with haplogroup $T$ have a lower activity of respiratory chain complexes than sperm cells with haplogroup H [5]. In contrast, another study was not able to demonstrate bioenergetic differences of isogenetic cell lines representing haplogroup $\mathrm{H}$ and $\mathrm{T}$ [27]. Unfortunately alteration of ROS production in these cell lines with different haplogroups has not been reported. We assume that the differences in ROS production among the haplogroups are subtle, requiring both time and interactions with other genetic and environmental factors before pathological manifestation. In accordance with previous studies, we found no association between mtDNA haplogroups and type 2 diabetes mellitus when all patients with diabetes (with and without retinopathy) were included [28]. Epidemiological studies in Japan showed an association of a mtDNA variation (position 5178, haplogroup N9b) with myocardial infarction $[29,30]$. We did not investigate the mtDNA variant located at position 5178 , which is an indicator for Asian haplogroup D and the N9b haplogroup, because these variations are not prevalent in European Caucasians. Our data, however, support the concept that mtDNA variability is associated with vascular diseases.

A recent study from Denmark found no association of mtDNA haplogroups with ischemic cardiovascular disease, cancer, infectious diseases, longevity and mortality in a general population of northern European descent [31]. The study was perspective, included a large number of individuals with a long follow up. No major association to a wide variety of diseases was observed, although 
there is a vast literature on the association of mtDNA haplogroups with several diseases available. One explanation could be the different geographical distribution of mtDNA haplogroups. Therefore, our study on a middle European population can not be directly compared with the Danish study. Furthermore, Benn et al. investigated cases with myocardial infarction or symptoms considered characteristic of angina pectoris whereas in our study we included patients with angiographically documented CAD (i.e. $\geq 50 \%$ stenosis of at least one of the major epicadial vessels) - a criteria consistently used in the literature defining clinically relevant CAD [32,33].

We assume that a certain percentage of our control subjects will develop CAD and/or diabetes. This might have led to an underestimation of the effects we observed in the present study. If these persons could have been excluded, then one could expect that the prevalence of haplogroup $\mathrm{T}$ would have been even lower than was observed in the present control cohort; this in turn would increase the statistical significance of our findings. When we performed statistical analysis of CAD patients with a similar mean of age as controls, we found that there was an even larger difference in frequencies of haplogroup $\mathrm{T}$ between cases and controls (data not shown). We can not exclude that other unknown and unmeasured confounding factors are present.

\section{Conclusion}

In the present study, mitochondrial haplogroup $\mathrm{T}$ is significantly associated with the presence of CAD and diabetic retinopathy, indicating that the presence of haplogroup $\mathrm{T}$ contributes to the risk of developing both $\mathrm{CAD}$ and diabetic retinopathy. The association between vascular disease and haplogroup variation of mtDNA, as demonstrated in the present study, should stimulate further investigation on their biological effects as well as on their relations to chronic diseases.

\section{Competing interests}

The authors declare that they have no competing interests.

\section{Authors' contributions}

$\mathrm{BK}, \mathrm{WS}, \mathrm{RM}$ designed the study. Patients were recruited by OS, IA, RM, MW, AH, RW, OS, BP, BI and WR. Laboratory work was undertaken by EEM, FAZ, DS, JAM and MW. Statistical analysis was done by WE and EEM. BK wrote the first draft of the paper. All other authors contributed to the final version of the manuscript and have approved the final version.

\section{Acknowledgements}

We thank Eva Schwaiger, Katharina Hammerl and Gabriele Kronberger for technical assistance.

The project was supported by the "Medizinische Forschungsgesellschaft Salzburg" and by a grant of the "Kamillo-Eisner Stiftung" to B. Paulweber.

\section{References}

I. Gutierrez J, Ballinger SW, Darley-Usmar VM, Landar A: Free radicals, mitochondria, and oxidized lipids: the emerging role in signal transduction in vascular cells. Circ Res 2006, 99:924-932.

2. DiMauro S, Schon EA: Mitochondrial respiratory-chain diseases. N Engl J Med 2003, 348:2656-2668.

3. Wallace DC: A mitochondrial paradigm of metabolic and degenerative diseases, aging, and cancer: a dawn for evolutionary medicine. Annu Rev Genet 2005, 39:359-407.

4. Torroni A, Petrozzi M, D'Urbano L, Sellitto D, Zeviani M, Carrara F, Carducci C, Leuzzi V, Carelli V, Barboni P, De Negri A, Scozzari R: Haplotype and phylogenetic analyses suggest that one European-specific mtDNA background plays a role in the expression of Leber hereditary optic neuropathy by increasing the penetrance of the primary mutations II 778 and I4484. Am J Hum Genet 1997, 60:1107-1121.

5. Ruiz-Pesini E, Lapena AC, Diez-Sanchez C, Perez-Martos A, Montoya J, Alvarez E, Diaz M, Urries A, Montoro L, Lopez-Perez MJ, Enriquez A: Human mtDNA haplogroups associated with high or reduced spermatozoa motility. Am J Hum Genet 2000, 67:682-696.

6. Esposito LA, Melov S, Panov A, Cottrell BA, Wallace DC: Mitochondrial disease in mouse results in increased oxidative stress. Proc Natl Acad Sci USA 1999, 96:4820-4825.

7. Rose G, Passarino G, Carrieri G, Altomare K, Greco V, Bertolini S, Bonafe M, Franceschi C, De Benedictis G: Paradoxes in longevity: sequence analysis of mtDNA haplogroup $J$ in centenarians. EurJ Hum Genet 200I, 9:70I-707.

8. Darvishi K, Sharma S, Bhat AK, Rai E, Bamezai RN: Mitochondrial DNA GI0398A polymorphism imparts maternal Haplogroup $\mathbf{N}$ a risk for breast and esophageal cancer. Cancer Lett 2007, 249:249-255.

9. Pyle A, Foltynie T, Tiangyou W, Lambert C, Keers SM, Allcock LM, Davison J, Lewis SJ, Perry RH, Barker R, Burn DJ, Chinnery PF: Mitochondrial DNA haplogroup cluster UKJT reduces the risk of PD. Ann Neurol 2005, 57:564-567.

10. Aiello LP, Avery RL, Arrigg PG, Keyt BA, Jampel HD, Shah ST, Pasquale LR, Thieme H, Iwamoto MA, Park JE, et al:: Vascular endothelial growth factor in ocular fluid of patients with diabetic retinopathy and other retinal disorders. N Engl J Med 1994, 33 I : | 480- I 487.

II. Aiello LP, Gardner TW, King GL, Blankenship G, Cavallerano JD, Ferris FL 3rd, Klein R: Diabetic retinopathy. Diabetes Care 1998, 2I:143-I56.

12. Norgaz T, Hobikoglu G, Aksu H, Guveli A, Aksoy S, Ozer O, Bolca $O$, Narin A: Retinopathy is related to the angiographically detected severity and extent of coronary artery disease in patients with type $\mathbf{2}$ diabetes mellitus. Int Heart J 2005, 46:639-646.

13. Wong TY, Klein R, Sharrett AR, Manolio TA, Hubbard LD, Marino EK, Kuller L, Burke G, Tracy RP, Polak JF, Gottdiener JS, Siscovick DS: The prevalence and risk factors of retinal microvascular abnormalities in older persons: The Cardiovascular Health Study. Ophthalmology 2003, I I 0:658-666.

14. Brownlee M: The pathobiology of diabetic complications: a unifying mechanism. Diabetes 2005, 54: I6I5-1625.

15. Madamanchi NR, Runge MS: Mitochondrial dysfunction in atherosclerosis. Circ Res 2007, 100:460-473.

16. Weitgasser R, Galvan G, Malaimare L, Derflinger I, Hedegger M, Lang J, Iglseder B, Ladurner G, Paulweber B: Cholesteryl ester transfer protein TaqIB polymorphism and its relation to parameters of the insulin resistance syndrome in an Austrian cohort. Biomed Pharmacother 2004, 58:619-627.

17. Wiesbauer M, Meierhofer D, Mayr JA, Sperl W, Paulweber B, Kofler $B$ : Multiplex primer extension analysis for rapid detection of major European mitochondrial haplogroups. Electrophoresis 2006, 27:3864-3868.

18. Malyarchuk BA, Grzybowski T, Derenko MV, Czarny J, Drobnic K, Miscicka-Sliwka D: Mitochondrial DNA variability in Bosnians and Slovenians. Ann Hum Genet 2003, 67:412-425.

19. Monsalve M, Borniquel S, Valle I, Lamas S: Mitochondrial dysfunction in human pathologies. Front Biosci 2007, I 2: I I 3 |- | I53.

20. Tan AL, Forbes JM, Cooper ME: AGE, RAGE, and ROS in diabetic nephropathy. Semin Nephrol 2007, 27:I30-| 43.

21. Schleicher E, Friess U: Oxidative stress, AGE, and atherosclerosis. Kidney Int Suppl 2007:SI 7-26. 
22. Raule N, Sevini F, Santoro A, Altilia S, Franceschi C: Association studies on human mitochondrial DNA: methodological aspects and results in the most common age-related diseases. Mitochondrion 2007, 7:29-38.

23. Duval C, Cantero AV, Auge N, Mabile L, Thiers JC, Negre-Salvayre A, Salvayre R: Proliferation and wound healing of vascular cells trigger the generation of extracellular reactive oxygen species and LDL oxidation. Free Radic Biol Med 2003, 35:I589-I 598.

24. Kopprasch S, Pietzsch J, Graessler J: Validation of different chemilumigenic substrates for detecting extracellular generation of reactive oxygen species by phagocytes and endothelial cells. Luminescence 2003, 18:268-273.

25. Kalman B: Role of mitochondria in multiple sclerosis. Curr Neurol Neurosci Rep 2006, 6:244-252.

26. Pike DA: Phylogenetic networks for human mtDNA haplogroup T. J of Genetic Genealogy 2006, 2: I-I I.

27. Amo T, Yadava N, Oh R, Nicholls DG, Brand MD: Experimental assessment of bioenergetic differences caused by the common European mitochondrial DNA haplogroups $\mathbf{H}$ and $\mathbf{T}$. Gene 2008, 41 I :69-76.

28. Chinnery PF, Mowbray C, Patel SK, Elson JL, Sampson M, Hitman GA McCarthy MI, Hattersley AT, Walker M: Mitochondrial DNA haplogroups and type 2 diabetes: a study of 897 cases and 1010 controls. J Med Genet 2007, 44:e80.

29. Takagi K, Yamada Y, Gong JS, Sone T, Yokota M, Tanaka M: Association of a 5I78C-->A (Leu237Met) polymorphism in the mitochondrial DNA with a low prevalence of myocardial infarction in Japanese individuals. Atherosclerosis 2004 I 75:28I-286.

30. Nishigaki Y, Yamada Y, Fuku N, Matsuo H, Segawa T, Watanabe S, Kato K, Yokoi K, Yamaguchi S, Nozawa Y, Tanaka M: Mitochondrial haplogroup $\mathbf{N} 9 b$ is protective against myocardial infarction in Japanese males. Hum Genet 2007, I 20:827-836.

31. Benn M, Schwartz M, Nordestgaard BG, Tybjaerg-Hansen A: Mitochondrial haplogroups: ischemic cardiovascular disease, other diseases, mortality, and longevity in the general population. Circulation 2008, I I 7:2492-250I.

32. Lenzen MJ, Boersma E, Bertrand ME, Maier W, Moris C, Piscione F, Sechtem U, Stahle E, Widimsky P, de Jaegere P, Scholte op Reimer WJ, Mercado N, Wijns W: Management and outcome of patients with established coronary artery disease: the Euro Heart Survey on coronary revascularization. Eur Heart J 2005, 26: I 169-I I79.

33. Do D, West JA, Morise A, Atwood E, Froelicher V: A consensus approach to diagnosing coronary artery disease based on clinical and exercise test data. Chest I997, I I I: I742-I749.

\section{Pre-publication history}

The pre-publication history for this paper can be accessed here:

http://www.biomedcentral.com/1471-2350/10/35/pre pub

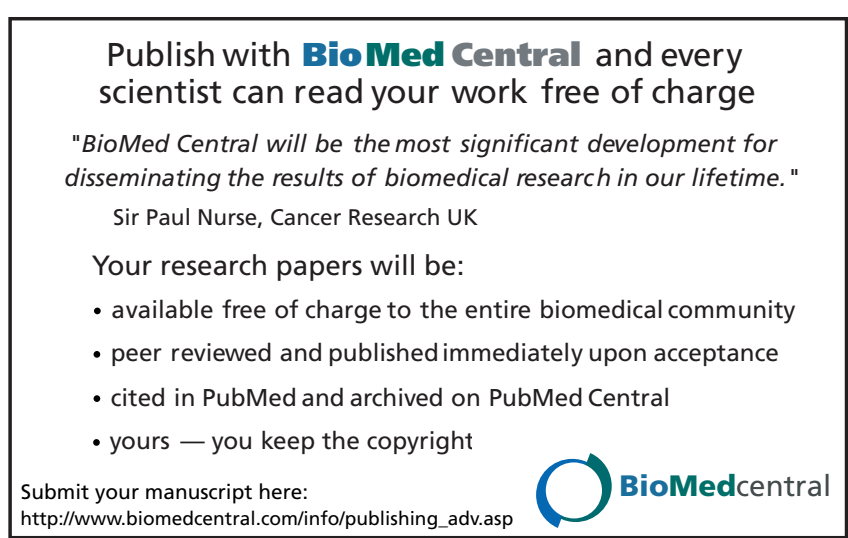

